\title{
Stromal changes in leukaemic and related bone marrow proliferations
}

\author{
N. G. SANERKIN \\ From the Department of Pathology, St. David's Hospital, \\ Cardiff, Wales
}

SYNOPSIS The stromal structure of the bone marrow was studied in 96 cases of leukaemia and related disorders.

The reticulin stroma forms an integral component of any given marrow proliferation and may often be increased in amount, sometimes normal, and occasionally decreased. The pattern is often indeterminate but certain distinguishable patterns may be found, including a sinusoidal pattern in myelofibrotic chronic myeloses, a pattern characterized by an abundance of hyperplastic capillaries and arterioles often seen in lymphoid leukaemias, and one with irregular focal reticulin proliferation in many acute undifferentiated leukaemias.

Adventitious collagen fibres are demonstrable in about half of all myeloses, acute and chronic, leukaemic and aleukaemic, and may be diffuse or focal. They apparently develop from thickening and collapse and condensation of the basic reticulin network, not from any primary fibroblastic proliferation. The development of fibrosis does not denote an aetiological relationship between the conditions in which it occurs, since it appears to be a non-specific sequel to marrow exhaustion and marrow necrosis. There is no justification for the diagnosis of acute or 'malignant' myelofibrosis as a disease entity in cases of acute leukaemia with marrow fibrosis.

Osteolytic change due to resorption of bony trabeculae is a common event in all the conditions studied and may be accompanied by remoulding of the eroded trabeculae. Fibre bone formation is found in about $15 \%$ of cases, including acute lymphoblastic leukaemias and acute undifferentiated leukaemias, but is extensive and well-developed only in cases of primary myelofibrosis.

An increase in marrow reticulin has long been recognized in primary myelofibrosis, but until recently (Burston and Pinniger, 1963) little was known about the reticulin structure of leukaemic marrows despite Masugi's contribution in 1926. Following a paper by Hutt, Smith, Clark, and Pinniger (1952) on the value of rib biopsy in the diagnosis of marrow disorders, there has been a tendency to accept an increase in marrow reticulin as evidence of myelofibrosis. Similarly, there has been a tendency to diagnose as 'acute myelofibrosis' cases of acute leukaemia with brisk reticulin and early collagen production. Two such cases were so diagnosed in our own necropsy records, and such a diagnosis may be further encouraged by the recent paper by Lewis and Szur (1963) who described 'malignant myelosclerosis' as a disease entity, an acute variant of 'chronic myelosclerosis'.

This study was undertaken to determine the Received for publication 16 December 1963. incidence of stromal changes in the bone marrow in leukaemias and related disorders, in an effort to assess their significance in relation to the problem of myelofibrosis.

\section{MATERIAL}

In the necropsy records of the Department of Pathology, University of Bristol, 96 cases of leukaemia, polycythaemia vera, and primary myelofibrosis were found with suitable blocks from the bone marrow. These included 17 cases of acute myeloblastic leukaemia, 19 of acute undifferentiated leucoblastic leukaemia, 21 of acute lymphoblastic leukaemia, one of chloroma (chloromyeloleukaemia), five of monocytic leukaemia, 10 of chronic myelocytic leukaemia, 10 of chronic lymphocytic leukaemia, five of polycythaemia vera, and eight of primary myelofibrosis.

Sections at $5 \mu$ were stained for collagen fibres 
with haematoxylin and Van Gieson's stain and for reticulin fibrils by Gordon and Sweet's silver impregnation method.

\section{DEFINITIONS}

In this paper the term 'myelofibrosis' is used to denote the presence of adventitious fibrous tissue in the bone marrow, whether diffuse or focal, 'primary', or secondary. The term 'primary myelofibrosis' is used in the accepted manner to describe the clinicopathological syndrome in which there is slowly progressive splenomegaly, a leucoerythroblastic blood picture, and replacement of bone marrow by fibrous tissue with or without osseous proliferation.

It is proposed to employ the term 'myelophthisis' or 'marrow exhaustion' to describe haemopoietic cell depletion or depopulation in a previously active marrow.

'Myelonecrosis' is used as defined by Peace (1953) to describe identifiable necrosis of haemopoietic cells.

\section{RESULTS}

MARROW CELlULARITY Varying degrees of leukaemic cell depletion or myelophthisis can be seen in a substantial majority of these conditions. It may be focal and patchy, as often in the acute leukaemias, or may tend to be extensive and diffuse as in cases of chronic myelocytic leukaemia, polycythaemia vera, and primary myelofibrosis. Chronic myelophthisic states, whatever the nature of the antecedent marrow proliferation, showed a consistent picture with hypocellularity and fibrosis. A variable degree of myelophthisis and occasionally of myelonecrosis was present in acute leukaemias, most prominently in undifferentiated ones, in many of which it was associated with the development of young fibrous tissue.

In areas of marrow fibrosis, focal or diffuse, it was difficult to identify typical fibroblasts with any degree of certainty. Cells with elongated or rod-like nuclei were often scattered about in such areas but they could well be reticulo-endothelial rather than fibroblastic in nature.

RETICULIN FIBRILS An increase in reticulin fibrils can be found in all these conditions. A very brisk increase was present in all cases of primary myelofibrosis (Fig. 1), in some cases of chronic myelocytic leukaemia (Fig. 2) and polycythaemia vera, and in some acute leukaemias (Figs. 3, 4, 5). Lesser degrees of reticulin increase, varying from slight to considerable, were commonly present in most others, although in some cases the reticulin was normal and in a few definitely decreased in amount.
A basic stellate reticulin network, whether $\Rightarrow$ increased in amount or not, would appear to be anঙ intrinsic component of all these proliferations. The? component fibrils may be fine or coarse. Coarse 흘 reticulin fibrils have been described as 'fibroblastic' by Burston and Pinniger (1963) since they represent ${ }^{\mathbb{D}}$ immature collagen fibres still retaining their argyrophilia. Although such coarse fibrils are an invariable $\rightarrow$ feature of chronic myelofibrotic marrows (Figs. 1, 2),, they may also be found in fairly cellular leukaemias $\vec{\omega}$ (Figs. 4, 5) showing only occasional random fine collagen fibres, and may be absent in foci of acute? myelophthisis with fibrosis. These coarse argyrophilic fibrils and the collagen fibres resulting there-ir from appear to develop from a progressive thickeninger of the basic reticulin network and not from any $\rightarrow$ fibroblastic activity. In leukaemias with focalo myelophthisis and fibrosis, the basic reticulinnetwork, whether fine or coarse, loses its stellate $\mathcal{\Phi}^{\infty}$ arrangement, its fibrils become condensed, approxi-O mated, wavy and parallel to one another (Figs. 5, 6). $\frac{\mathbb{D}}{3}$ Simultaneously, an extremely fine tufted closely-o knit meshwork may appear between the collapsed fibrils, cementing the latter to one another. This isce probably due to condensation of the finest reticulin + fibrils which may not ordinarily be discerniblep before collapse and condensation. These alterations in the reticulin network are clearly a passive sequel to the partial disappearance of pre-existing leukaemic cells from the interstices of the basic reticulin framework.

The reticulin pattern in these conditions tends too be indeterminate with certain interesting exceptions. In primary myelofibrosis and in many cases of chronic myelocytic leukaemia and polycythaemia vera, a remarkable feature is not merely the brisk increase in reticulin fibrils but the presence of $a-$ prominent sinusoidal vascular pattern (Figs. 1, 8), although this may become less obtrusive in occa-o sional cases showing advanced fibrosis. A rather distinctive reticulin pattern is often seen in lymphoidg leukaemias, both acute and chronic, and sometimess in acute non-lymphoid leukaemias as well, in which? multiple, tiny, punched-out holes are interspersec among the reticulin fibrils (Fig. 7), reflecting an abundance of hyperplastic capillaries and arterioles $N$ A patchy irregular distribution of reticulin, whertw found, is highly suggestive of acute undifferentiated leukaemia since in many of these the reticulin fibrils like the leukaemic tissue, may be confined to irregular foci.

COLLAGEN FIBRES Adventitious collagen fibres can be found in over $50 \%$ of these cases. Fibrous tissue tends to be diffuse and extensive in all cases of primary myelofibrosis and in many cases of chronige 

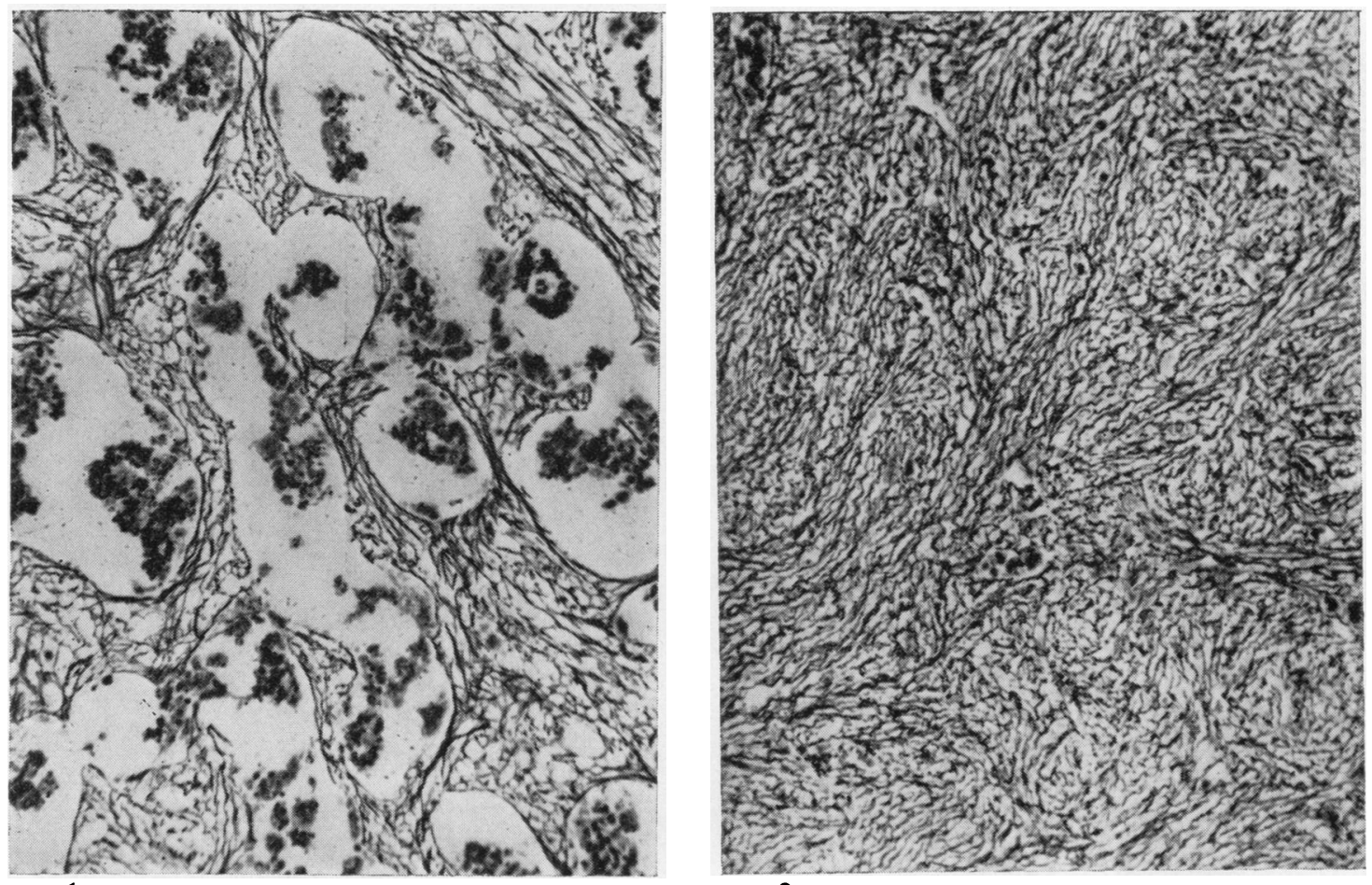

FIG. 1 .

FIG. 2.

FIG. 1. Reticulin pattern in primary myelofibrosis. Brisk reticulin production; prominent sinusoidal vascular system. Silver impregnation for reticulin. $\times 120$.

FIG. 2. Brisk reticulin formation in a case of chronic myelotic leukaemia with advanced fibrosis; sinusoidal system largely obliterated. Retic. $\times 120$.



FIG. 3 .

FIG. 3. Focal reticulin proliferation in a case of acute undifferentiated leukaemia. Retic. $\times 120$.

FIG. 4. Brisk reticulin formation in cellular area in chloroma. Compare Fig. 6 showing reticulin pattern in myelophthisic area in same case. Retic. $\times 475$. 


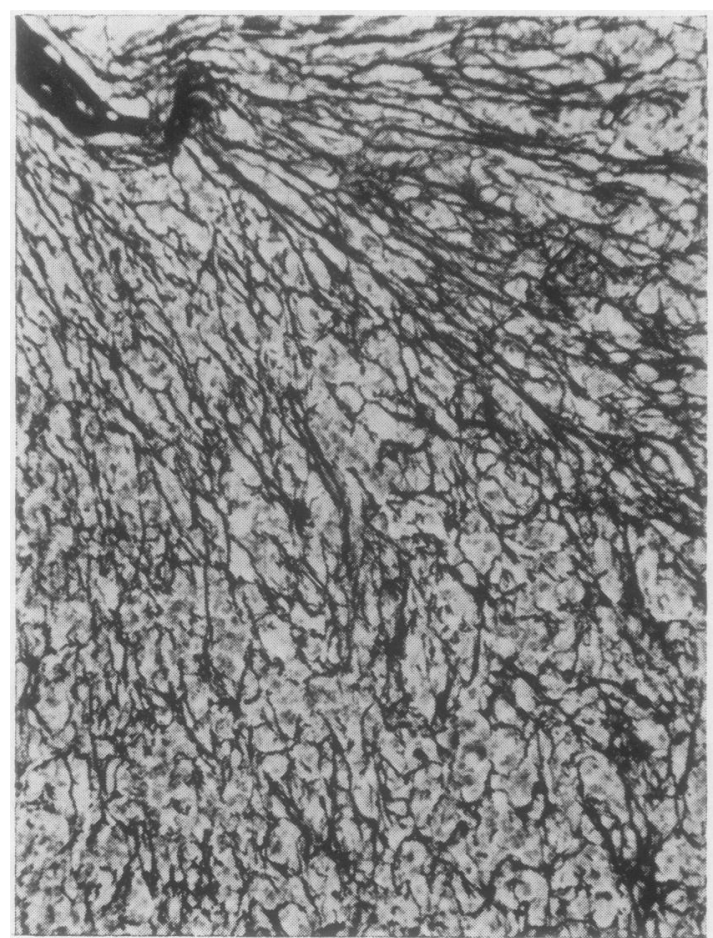

FIG. 5 .

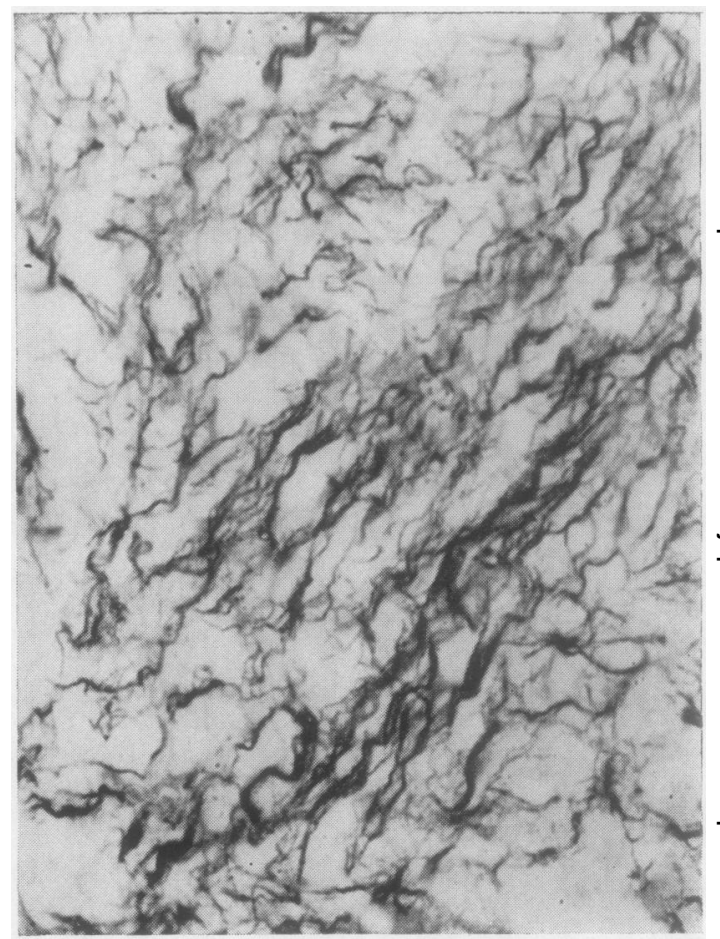

FIG. 6.

FIG. 5. Brisk reticulin formation in monocytic leukaemia. At the top is a zone with condensation and approximation of $₫$ reticulin fibrils and early fibrous tissue formation. Retic. $\times 120$.

FIG. 6. Reticulin pattern in myelophthisic area in chloroma, showing collapse and condensation of reticulin fibrils. Compare Fig. 4 showing reticulin pattern in cellular area in same case. Retic. $\times 475$.

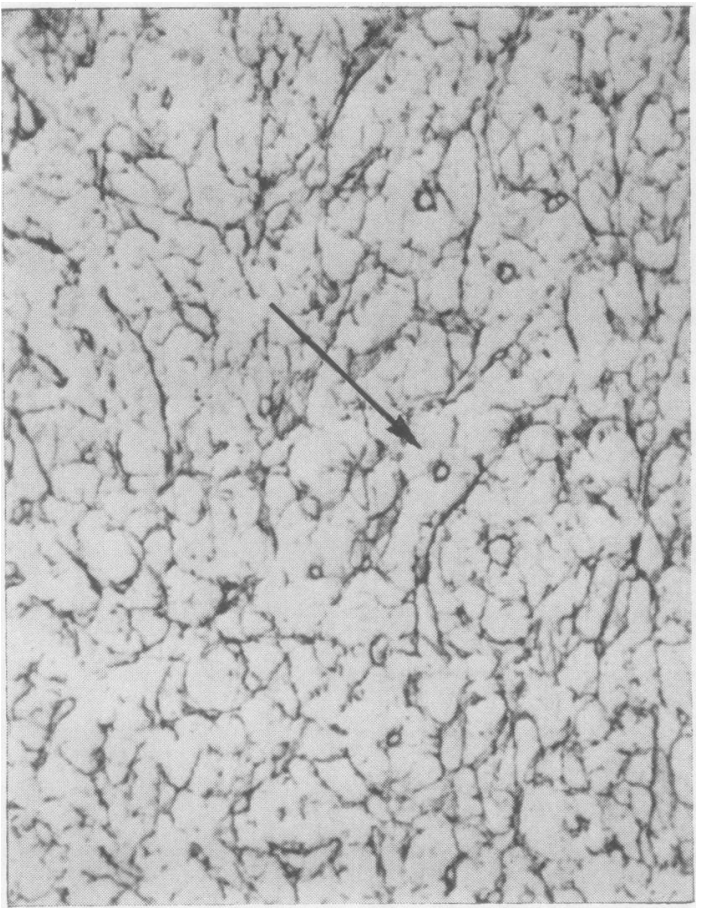

FIG. 7.

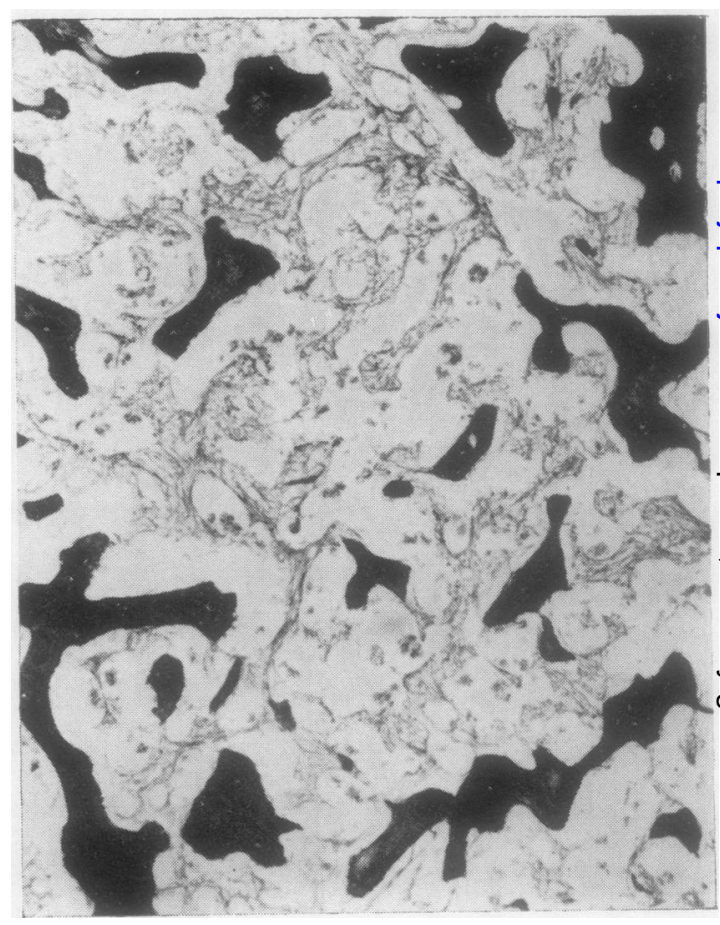

FIG. 8.

FIG. 7. Reticulin pattern common in lymphoid leukaemias, also seen in some acute leukaemias. Punched-out holes interspersed among the reticulin fibrils represent the abundant hyperplastic capillaries and arterioles. Retic. $\times 86$. FIG. 8. Abundant fibre bone formation in primary myelofibrosis. Note prominent sinusoidal vascular pattern. Retic. $\times 42 . \overline{0}$ 


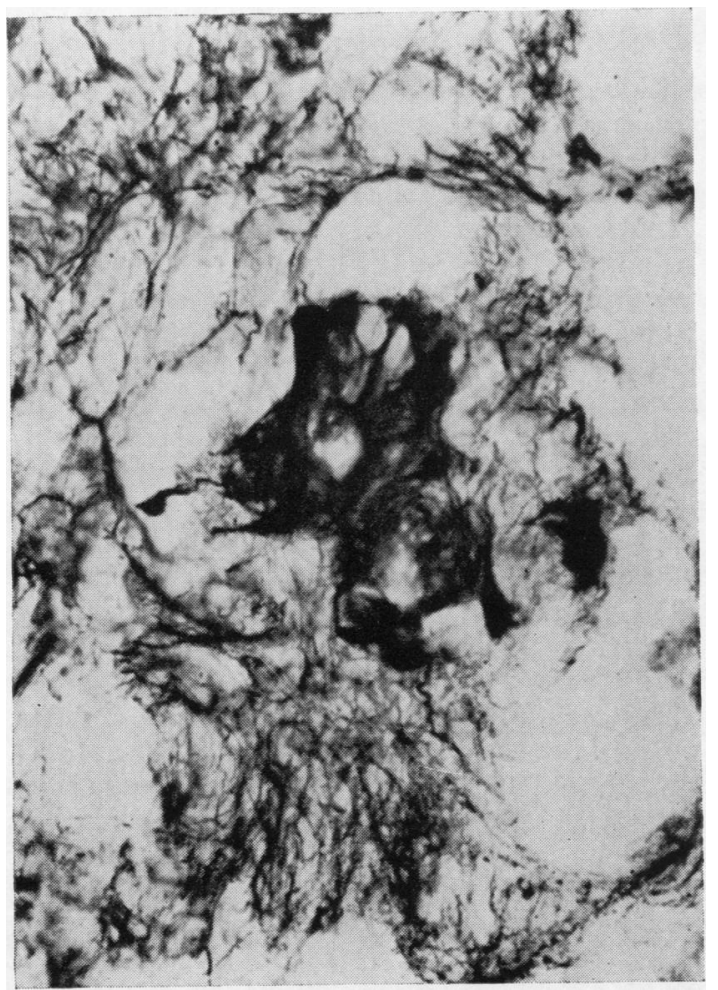

FIG. 9. Early fibre bone formation in a focus of myelophthisis and fibrosis in acute undifferentiated leukaemia. At the bottom there is a fine, closely-knit meshwork of reticulin fibrils with only occasional coarse reticulin strands. Retic. $\times 475$.

myelocytic leukaemia and polycythaemia vera. In these, the collagen fibres are arranged as confluent, elongated, fine, wavy fibres, usually running parallel to one another in the intersinusoidal tissue and linking up with the peritrabecular and periarterial fibrous tissue. In a few of these cases the fibrosis becomes very dense. Less prominent confluent fine fibrosis can be seen in many cases of acute leukaemia of all types as well as in chronic lymphocytic leukaemia. As mentioned in the account of reticulin fibrils, these elongated parallel wavy collagen fibres appear to develop from progressive thickening of the basic argyrophilic reticulum. Focal collagen formation occurs in foci of myelophthisis in many acute undifferentiated leukaemias, in which young collagen fibres tend to form an irregular, ill-defined, tangled skein (Fig. 9), and in many acute and chronic lymphoid leukaemias, in which collagen develops in discrete clumps, small and rounded, fairly sharply circumscribed. Somewhat similar circumscribed deposits of chunky collagen are occasionally found in cases of acute undifferentiated leukaemia.

The development of collagenous tissue appears to be directly proportional to the severity of marrow exhaustion. Where the exhaustion is uniform and diffuse, the fibrosis tends likewise to be diffuse; where exhaustion is focal, so is the fibrosis.

osseous CHANGES Osteolytic change due to resorption of bony trabeculae is a very frequent occurrence in all these disorders. Its existence in primary myelofibrosis was stressed by Vaughan and Harrison (1939). In many instances, this patchy trabecular resorption is accompanied by remoulding of the eroded trabeculae, sometimes producing a pattern simulating Paget's disease of bone.

Fibre bone is found in appreciable and sometimes excessive quantities in many cases of primary myelofibrosis (Fig. 8). Focal fibre bone formation (Fig. 9) is also seen in some cases of acute undifferentiated leukaemia, occasionally quite prominently, as well as in cases of acute lymphoblastic leukaemia in which it may develop in the isolated foci of chunky collagen already described. Fibre bone was significantly absent in cases of chronic myelocytic leukaemia and polycythaemia vera, despite the close similarities in the reticulin and collagenous structure of these conditions to that of primary myelofibrosis in which fibre bone formation is so common a feature.

VASCUlar CHANGes Prominent perivascular infiltration by leukaemic cells can be seen in most leukaemias, particularly in acute cases, often disrupting the adventitial collagen, remnants of which are displaced outwards and often undergo reactive sclerosis and thickening. Infiltration of the media is, however, a rare phenomenon seen in occasional cases only.

Hyperplastic capillaries and arterioles can be found in most leukaemias but are most abundant in acute leukaemias and in chronic lymphocytic leukaemia. The presence of a prominent sinusoidal system in primary myelofibrosis, chronic myelocytic leukaemia, and polycythaemia vera has been referred to already.

\section{DISCUSSION}

Masugi (1926) noted an increase in reticulin fibrils in cases of chronic myeloid leukaemia and chronic aleukaemic myelosis although not in acute leukaemias. Burston and Pinniger (1963) found increased reticulin in most of their leukaemias, including acute ones. The present paper broadly confirms their findings and fully concurs with their conclusion that 
the brisk production of reticulin does not of itself constitute sufficient evidence for making a diagnosis of myelofibrosis, although disagreeing with them on certain points of interpretation. They recognized two distinct patterns of reticulin, one said to be essentially normal since the fibrils of the network differed in no way from those of normal bone marrow, the other described as 'fibroblastic' due to the presence of immature collagen still retaining its argyrophilia. It does not, however, follow that the reticulin pattern in a leukaemic marrow must be 'normal' because its component fibrils are identical with those of normal marrow, any more than the reticulin pattern of a lymphosarcomatous lymph node is 'normal' because its fibrils may be individually indistinguishable from those of a normal lymph node. A basic reticulin network is present as an integral component of all leukaemic and related marrow proliferations and the only difference between fine and coarse reticulin fibrils is a qualitative one depending on fibril thickness, not one of of pattern. Burston and Pinniger (1963) also postulated that a fine reticulin network in the bone marrow was a physiological response whereby the amount of stroma varied directly with the amount of haemopoietic tissue. This is demonstrably not the case, since highly cellular marrows may have a normal or decreased reticulin content. A similar phenomenon is well recognized in reticulum-cell sarcomata which may have exceptionally brisk or very scanty reticulin (Marshall, 1956).

Fibrous tissue may develop in any of the leukaemias and related proliferations in inverse proportion to the cellularity of the marrow. The presence of a hypercellular marrow during the earlier stages of primary myelofibrosis has been established by many writers (including Jackson, Parker, and Lemon, 1940; Carpenter and Flory, 1941; Erf and Herbut, 1944; Heller, Lewisohn, and Palin, 1947; Merskey, 1949; Block and Jacobson, 1950; Wyatt and Sommers, 1950; Peace, 1953). As this hypercellular marrow becomes exhausted it also becomes correspondingly fibrotic. Two possible explanations may be put forth for this phenomenon. First, in the progression of the disease the primitive reticular cells, from which the marrow proliferation primarily derives, may lose their potentiality to produce haemopoietic cells and simultaneously revert to a fibrogenic role, one of the properties of reticular cells (Marshall, 1956), leading to increasing fibrosis. Such a process undoubtedly operates in many of the chronic myeloses, both leukaemic and aleukaemic. The other alternative (Wyatt and Sommers, 1950; Peace, 1953) postulates that the common denominator of all myelofibrotic diseases may be widespread necrosis of immature haemo- poietic cells leading to a reparative fibrosis. The $\frac{\stackrel{0}{*}}{\frac{0}{\sigma}}$ operation of such a mechanism in chronic myelo- $\underset{\Rightarrow}{\vec{F}}$ phthisic states could not be substantiated by the $\stackrel{\mathscr{P}}{\rightarrow}$ present study, since in such states actual cell necrosis $\bar{c}$ was impossible to demonstrate with any certainty, 흠 even though its possibility cannot entirely be ruled out. Its operation in acute leukaemias, however, $\stackrel{\square}{\unrhd}$ was amply confirmed and there can be little doubt that this type of post-necrotic fibrosis is not un- $\overrightarrow{0}$ common in acute leukaemias showing foci of myelophthisis or myelonecrosis. Since marrow fibrosis, even with development of fibre bone, may occur in acute leukaemias, there can be no justifi- 용 cation for the diagnosis of acute or 'malignant' $\vec{v}$ myelofibrosis (Lewis and Szur, 1963) solely upon ir demonstration of increased reticulin fibrils and 0 collagen fibres.

The presence of myelofibrosis does not signify an aetiological relationship between the conditions in which it occurs, since it appears to be a non-specific $\mathbb{D}$ sequel to marrow exhaustion and marrow necrosis. Similarities between chronic myelocytic leukaemia, polycythaemia vera, and chronic aleukaemic myelosis may well be fortuitous, and these conditions are probably distinct entities as maintained by Leonard, Israëls, and Wilkinson (1957) rather than intimately related disorders as suggested, among others, by Vaughan and Harrison (1939), Rosenthal and Erf (1943), Heller et al. (1947), and Dameshek (1951).

The possible role of treatment in the causation of marrow fibrosis has long been a source of controversy. Churg and Wachstein (1944) were inclined to discount the role of radiotherapy. Leonard et al. (1957), on the other hand, considered that marrow fibrosis in chronic myeloid leukaemia and polycythaemia vera was most probably due to irradiation. Burston and Pinniger (1963) could not find any correlation between their 'fibroblastic' cases and various forms of treatment. So many of the cases of leukaemia listed in the present series had received some form or other of treatment that it would be impossible accurately to assess their effect, if any, on the development of marrow fibrosis. The significance of myelophthisis and myelonecrosis has, however, been stressed in relation to such fibrosis, and any form of treatment may well accelerate if not initiate the process of myelophthisis and myelonecrosis.

It is a great pleasure to express my indebtedness to Professor T. F. Hewer, in whose Department this work was carried out, and to Dr. D. M. D. Evans for helpful criticism. My thanks are also due to Mr. T. A. Morris for technical assistance and to Mr. G. F. Haddock for help with the photography. 


\section{REFERENCES}

Block, M., and Jacobson, L. O. (1950). J. Amer. med. Ass., 143, 1390. Burston, J., and Pinniger, J. L. (1963). Brit. J. Haematol., 9, 172. Carpenter, C., and Flory, C. M. (1941). Arch. intern. Med., 67, 489. Churg, J., and Wachstein, M. (1944). Amer. J. med. Sci., 207, 141. Dameshek, W. (1951). Blood, 6, 372.

Erf, L. A., and Herbut, P. A. (1944). Ann. intern. Med., 21, 863. Heller, E. L., Lewisohn, M. G., and Palin, W. E. (1947). Amer. J. Path., 23, 327.

Hutt, M. S. R., Smith, P., Clark, A. E. and Pinniger, J. L. (1952). J. clin. Path., 5, 246.
Jackson, H. Jr., Parker, F. Jr., and Lemon, H. M. (1940). New Engl. J. Med., 222, 985.

Leonard, B. J., Israëls, M. C. G., and Wilkinson, J. F. (1957). Quart. J. Med., 26, 131.

Lewis, S. M., and Szur, L. (1963). Brit. med. J., 2, 472.

Marshall, A. H. E. (1956). An Outline of the Cytology and Pathology of the Reticular Tissue, pp. 7, 195-196. Oliver \& Boyd, Edinburgh \& London.

Masugi M. (1926). Jap. J. med. Sci., Trans. Path., $1,1$.

Merskey, C. (1949). Arch. intern. Med., 84, 277.

Peace, R. J. (1953). Amer. J. Path., 29, 1029.

Rosenthal, N., and Erf, L. A. (1943). Arch. intern. Med., 71, 793.

Vaughan, J. M., and Harrison, C. V. (1939). J. Path. Bact., 48, 339.

Wyatt, J. P., and Sommers, S. C. (1950). Blood, 5, 329.

\section{Broadsheets prepared by the Association of Clinical Pathologists}

The following broadsheets (new series) are published by the Association of Clinical Pathologists. They may be obtained from Dr. R. B. H. Tierney, Pathological Laboratory, Boutport Street, Barnstaple, N. Devon. The prices include postage, but airmail will be charged extra.

3 The Detection of Barbiturates in Blood, Cerebrospinal Fluid, Urine, and Stomach Contents. 1953. L. c. NICKOLLS. 1s.

4 The Estimation of Carbon Monoxide in Blood. 1953. D. A. STANLEY. $1 \mathrm{~s}$.

13 The Identification of Serotypes of Escherichia coli Associated with Infantile Gastro-enteritis. 1956. JOAN TAYLOR. $1 \mathrm{~s}$.

16 Preservation of Pathological Museum Specimens. 1957. L. W. PROGER. 1s.

17 Cultural Diagnosis of Whooping-cough. 1957. B. W. LACEY. 1s.

20 Investigation of Porphyrin/Porphyria. 1958 (reprinted 1962). C. RIMINGTON. 2s.

23 The Dried Disc Technique for Bacterial Sensitivity Tests. 1959. R. W. FAIRBROTHER and J. C. SHERRIS. 1s.

24 Safe Handling of Radioactive Tissues in the Laboratory and Post-mortem Room. 1959. R. C. CURRAN. 1s.

26 The Periodic Acid-Schiff Reaction. 1959. A. G. E. PEARSE. 1s.

28 Daily Fatty Acid Excretion. 1960. A. C. FRAZER. 2s.

29 The Preparation of Bone for Diagnostic Histology. 1960. D. H. Collins. $2 \mathrm{~s}$.

30 Control of Accuracy in Chemical Pathology. 1961. G. H. GRANT. 4s.

31 Investigation of Haemorrhagic States with Special Reference to Defects of Coagulation of the Blood. 1961. E. K. BLACKBURN. 4s.

32 Detection of Resistance to Streptomycin, P.A.S., and Isoniazid in Tubercle Bacilli. 1961. R. CRUICKSHANK and S. M. STEWART. 2 s.
33 The Laboratory Detection of Abnormal Haemoglobins. 1961. H. LEHMANN and J. A. M. AGER. 4s.

34 Titration of Antistreptolysin O. 1961. H. GOODER and R. E. O. WILLIAMS. 2s.

35 The Estimation of Faecal 'Urobilinogen'. 1961. C. H. GRAY. 2 s.

36 Quantitative Determination of Porphobilinogen and Porphyrins in Urine and Faeces. 1961. c. RMMINGTON. 3s. 6d.

37 The Paper Electrophoresis of Serum and Urinary Proteins. 1961. G. FRANGLen and N. H. MARTIN. 4s.

38 The Augmented Histamine Gastric Function Test. 1961. M. LUBRAN. $2 s$.

39 Investigation of Haemolytic Anaemia. 1961. J. G. SELWYN. 2 s.

40 Short-term Preservation of Bacterial Cultures. 1962. E. JOAN STOKES. 2s.

41 Serological Tests for Syphilis. 1962. A. E. WILkINsoN. 6s.

42 The Determination of Glucose 6-Phosphate Dehydrogenase in Red Cells. 1962. T. A. J. PRANKERD. 2s.

43 Mycological Techniques. 1962. R. W. RIDDELL. 3s. 6d.

44 The Laboratory Investigation of Catecholamine Secreting Tumours. 1963. M. SANDLER and C. R. J. RUTHVEN. 2s.

45 Diagnostic Test for Hereditary Galactosaemia. 1963. v. SCHWARZ. 2 s.

46 The Determination of Serum Iron and Total Iron Binding Capacity. 1963. A. JORDAN and D. A. PODMORE. 2s.

47 Nuclear Sexing. 1964. B. LENNOX and w. M. DAVIDSON. $2 \mathrm{~s}$. 